\title{
WEBTEACH: WEB TOOLS FOR TEACHERS AND STUDENTS
}

\author{
Franco Bagnoli $^{1}$, Fabio Franci ${ }^{2}$ and Andrea Sterbini ${ }^{3}$
}

\begin{abstract}
We present the WebTeach system, currently used at the Engineering Faculty, University of Florence, Italy. The system is composed by three parts: WebCheck, a web database interface for test management; WebTest, a multiple-choice quiz generation system and WebWrite, a collaborative web publishing tool. WebCheck is used for managing subscriptions to tests and publication of results. It allows teachers to organize exam sessions and students to consult the results of their tests by a web interface. Most of tests are generated using WebTest, which is a suite of perl libraries and front-end applications for the generation and automatic evaluation of multiple-choice quizzes. WebWrite is an implementation of the TWiki system, which appears as a web site where all pages can be edited using a simple syntax, resembling e-mail text. WebWrite is used for publishing didactic material and will become the generic interface-managing tool of the WebTeach system.

This system is also used for teaching to jailed students.
\end{abstract}

Index Terms — Distance learning, Web-based tools.

\section{The WeB Teach ProjeCt}

The WebTeach project started three years ago as a small online system to manage students scheduling for examinations. At present it has grown to include three different tools — WebCheck, WebWrite, WebTest — and enhancements are continuosly being added.

The WebCheck tool is based on a set of perl [1] scripts interacting with an sql database [2]. After having asked to an administrator to be registered into the system, teachers have to activate a course for a given academic year, activate a test for a given course, and eventually publish the results for a given test. Teachers can also extract cumulative reports for each course.

Students can register themselves into the system using the unique numeric id given by the University of Florence. After authentication, each student can edit his/her personal data, register for a test, or check the corresponding results.

WebWrite is an implementation of the TWiki system [3] (a particular Wiki Wiki Web [4]), which is a cooperative environment for editing web pages.

Page contents are stored as text files, allowing the use of normal UNIX tools like grep to perform searches, RCS for version control and so on. The formatting elements are kept at minimum, like in email text. The rendering of HTML is done automatically by the system. All pages can have files attached, simplifying the distribution of ddactic material. Moreover, teachers exploit WebWrite to encourage debates among students in the form of threaded discussion.

Thanks to its free-software approach, we have designed and realized new plugins to improve the usability of WebWrite, like managing LaTeX fragments in the topic text, including $2 \mathrm{D}$ and $3 \mathrm{D}$ plots and a calendar of important events.

WebTest was realized to handle random generation and automatic correction of multiple-choice quizzes.

The quiz database is a LaTeX file with special tags marking the beginning of group of homogeneous questions, of questions themselves, of right and wrong answers. The actual test is defined by a specification file, which is a text file specifying the total number of different tests, the number of questions to be sampled from each group, and the weight to be assigned to right, blank and wrong answers.

The answers to the tests are specified using a string of characters, which has to be copied on a text file by teachers. Marks are computed and optionally inserted into the WebCheck database in a semi-automatic way. It is already possible to use WebWrite for sharing the database of questions among teachers. We are working to use it also as a graphical front-end to the WebTest suite.

\section{Survey of WebTeach usage}

WebCheck is used by more than 70 teachers for about 130 courses, 450 tests (each with an average participation of 25 students), and a total of about 4500 students.

A previous version of the WebWrite tool was used to produce documentation for three courses (computer science, advanced geometry and calculus) and as a virtual lab of dynamical systems and statistical mechanics. This material is being migrated to the present version (which uses the new plugins described above) together with new material in the Physics field.

The WebTest tool is currently used by more than 20 teachers, and served for the entry test to all freshmen in the Florentine Engineering faculty (about 1000 tests).

\section{REFERENCES}

[1] Perl: http://www.perl.org

[2] MySQL: http://www.mysql.org

[3] TWiki: http://www.TWiki.org

[4] Leuf, B. and Cunningham, W., "The Wiki Way: quick collaboration on the Web", Addison Wesley 2001

\footnotetext{
${ }^{1}$ Franco Bagnoli, Dipartimento di Energetica, University of Florence, Via S. Marta, 3 I-50139 Florence, Italy, bagnoli@dma.unifi.it

${ }^{2}$ Fabio Franci, Dipartimento di Sistemi e Informatica, University of Florence, Via S. Marta, 3 I-50139 Florence, Italy, fabio@dma.unifi.it

${ }^{3}$ Andrea Sterbini, Dipartimento di Scienze dell'Informazione, University of Rome 1, via Salaria, 113 I-00198 Rome, Italy, sterbini@dsi.uniroma1.it 